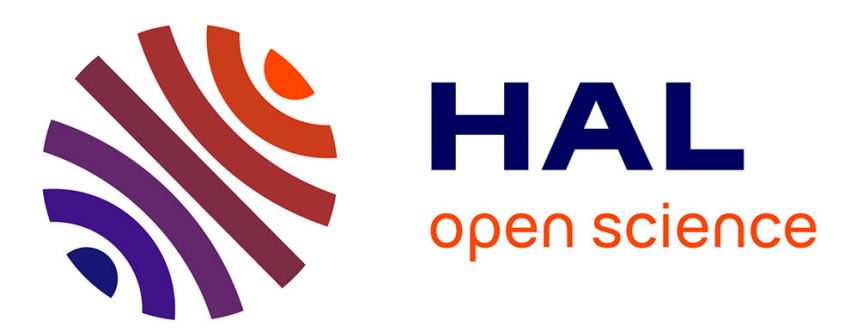

\title{
Influence de l'état sanitaire des populations du passé sur la mortalité en temps de peste: contribution à la paléoépidémiologie
}

\author{
Sacha Kacki
}

\section{- To cite this version:}

Sacha Kacki. Influence de l'état sanitaire des populations du passé sur la mortalité en temps de peste: contribution à la paléoépidémiologie. Bulletins et Mémoires de la Société d'anthropologie de Paris, 2017, 29 (3-4), pp.202-212. hal-03140734

\author{
HAL Id: hal-03140734 \\ https://hal.science/hal-03140734
}

Submitted on 13 Feb 2021

HAL is a multi-disciplinary open access archive for the deposit and dissemination of scientific research documents, whether they are published or not. The documents may come from teaching and research institutions in France or abroad, or from public or private research centers.
L'archive ouverte pluridisciplinaire HAL, est destinée au dépôt et à la diffusion de documents scientifiques de niveau recherche, publiés ou non, émanant des établissements d'enseignement et de recherche français ou étrangers, des laboratoires publics ou privés. 
Influence de l'état sanitaire des populations du passé sur la mortalité en temps de peste : contribution à la paléoépidémiologie

Investigating the relation between health status and plague mortality in past populations: a contribution to paleoepidemiology

\section{Sacha KACKI}

PACEA, UMR 5199, Université de Bordeaux, Bât. B8 allée Geoffroy Saint-Hilaire, 33615 Pessac cedex, France. sacha.kacki@gmail.com

Résumé : Les épidémies de peste qui ont régulièrement frappé l'Europe depuis le VIe siècle de notre ère, bien que largement documentées par les sources historiques, restent encore mal connues du point de vue épidémiologique. Une incertitude demeure notamment quant à savoir si la maladie cibla préférentiellement certaines catégories de population ou entraîna au contraire une mortalité uniforme. Le présent travail contribue à cette discussion par le prisme de l'étude anthropologique et paléopathologique d'un large corpus de squelettes médiévaux et modernes $(\mathrm{N}=1090)$ issus de quatre sites d'inhumation de pestiférés et de deux cimetières paroissiaux utilisés hors contexte épidémique. Les résultats révèlent une signature démographique commune aux séries de peste, qui s'avère distincte d'un modèle de mortalité naturelle, mais en adéquation avec la structure d'une population vivante préindustrielle. L'analyse de divers indicateurs de stress suggère par ailleurs que les victimes de la peste jouissaient d'un meilleur état de santé préexistant que les individus morts d'autres causes. Les résultats tendent à prouver que les facteurs causaux de ces lésions, d'accoutumé responsables d'une diminution des chances de survie, n'eurent qu'une faible influence sur le risque de mourir de l'infection pesteuse. L'étude démontre in fine que la peste fut par le passé à l'origine d'une mortalité non sélective, frappant indistinctement les personnes des deux sexes, de tout âge et de toute condition sanitaire.

Mots-clefs : épidémies, Peste noire, bioarchéologie, démographie, paléopathologie, sélectivité. 
Abstract: From the sixth century, plague caused recurrent mortality crises in the Western world. Although some aspects of the history of plague epidemics has now become well known, still many unanswered questions exist, such as the precise epidemiological pattern of the disease in the ancient times. In particular, it is unclear that whether plague has killed people indiscriminately or it has been selective. The research contributed to the debate through an anthropological and palaeopathological study of the skeletal remains of 1090 individuals inclusive of plague victims from four medieval and post-medieval burial grounds and individuals from two parish cemeteries in use during normal mortality periods. Our results reveal a common demographic signature for the plague-related assemblages, which were different against the pattern for natural mortality but closely matched with the demographic structure of a living preindustrial population. Moreover, our study of different non-specific skeletal stress markers showed that plague victims were in better health before they died when compared to the people who had died from other causes. The results suggest that individuals who suffered from stress and disease had fewer chances of survival in nonepidemic periods, whereas they were not at a higher risk of dying during plague epidemics. Our study thus provides evidence that plague was not selective, and that it killed regardless of sex, age and pre-existing health.

Keywords: epidemics, Black Death, bioarchaeology, demography, paleopathology, selectivity.

\section{Introduction}

Si la peste n'est de nos jours guère plus responsable que de quelques dizaines à quelques centaines de décès par an à l'échelle du globe [1,2], elle figura par le passé, et ce des siècles durant, au premier rang des maladies infectieuses les plus meurtrières. Causée par la bactérie Yersinia pestis, dont l'inoculation dans l'organisme humain s'opère le plus souvent par la piqûre de puces s'étant précédemment nourries sur des animaux infectés (en particulier certaines espèces de rongeurs) [3,4], cette zoonose se distingue par une extrême létalité en l'absence d'une antibiothérapie adaptée (40 à $70 \%$ de décès chez les individus souffrant de la forme bubonique de l'affection, $100 \%$ chez ceux développant une forme pulmonaire [5]). 
Cette caractéristique, couplée à la forte transmissibilité de la maladie et à sa grande célérité d'action, fit de la peste une cause majeure d'épidémies durant les périodes antérieures à l'avènement des thérapeutiques modernes. De récentes études paléogénomiques ont ainsi démontré qu'une souche ancestrale de la bactérie affectait déjà l'Homme à l'âge du Bronze [6,7]. Les ravages la maladie au sein des populations humaines sont toutefois plus particulièrement connus pour les périodes historiques [8-11], durant lesquels la peste est créditée d'avoir causé trois grandes pandémies (intervenues respectivement entre le VIe et le VIIIe siècle de notre ère, entre le milieu du XIVe siècle et la fin du XVIIIe siècle et à partir de la fin du XIXe siècle). L'unicité étiologique de ces vagues épidémiques, longtemps sujette à controverse [12], est désormais attestée par de multiples travaux de paléogénétique ayant permis l'identification répétée de séquences d'ADN spécifiques au bacille pesteux dans les restes squelettiques de victimes des première et deuxième pandémies [13-18].

L'importance qu'ont eue les épidémies de peste dans l'histoire de la civilisation européenne en a naturellement fait, depuis des décennies, le centre d'attention d'historiens, de sociologues et de démographes. Leurs travaux respectifs, fondés sur l'exploitation d'une riche documentation textuelle, ont permis d'acquérir une connaissance approfondie de diverses facettes de ces phénomènes épidémiques, concernant notamment leur chronologie et leur dynamique d'extension territoriale [7,10], leurs répercussions démographiques [19-21] et socio-économiques [11,22,23], ainsi que les moyens mis en œuvre par les populations pour tenter d'endiguer leur propagation [24-26]. Pour autant, des zones d'ombre subsistent sur certains aspects de ces épidémies, notamment sur leurs caractéristiques épidémiologiques. Si la virulence de la maladie est indéniable - preuve en est le décès de plus d'un quart de la population européenne durant la célèbre Peste noire (1347-1352) [10,27] -, une incertitude demeure quant à savoir si elle cibla préférentiellement certaines catégories de population ou entraîna au contraire une mortalité uniforme au sein des communautés affectées.

Les données épidémiologiques actuelles sont, à l'égard de cette question, relativement peu informatives, du fait de la relative rareté des cas de peste contemporains et de l'influence des mesures prophylactiques et curatives sur la marche naturelle des épidémies. Il en va de même des sources textuelles qui, se limitant pour les périodes antérieures au XVIIe siècle à des écrits narratifs, livrent des avis divergents sur les règles biologiques qui régirent la mortalité par peste. Ainsi, tandis que certaines rapportent que tous moururent également sans 
distinction selon l'âge, le sexe ou le statut socio-économique [28-30], d'autres prêtent à la maladie un caractère sélectif [31-33], lui attribuant toutefois variablement une prédilection pour les hommes ou pour les femmes, pour les enfants ou pour les personnes âgées, ou encore pour les pauvres ou pour les riches.

Face aux limites des sources écrites, l'étude anthropologique des séries de squelettes exhumés de cimetières de pestiférés apparaît à même d'apporter une contribution significative à la problématique, en cela qu'elle permet d'objectiver certaines des caractéristiques biologiques des victimes de la maladie. Force est toutefois de constater l'absence de consensus entre les précédentes études de telles collections. Aux arguments avancés par les partisans de la stricte non-sélectivité de la maladie [34-36] s'opposent en effet les résultats des travaux d'autres chercheurs, qui suggèrent que certains paramètres biologiques pourraient avoir eu une influence sur le risque de succomber à la peste (voir notamment les travaux de S. Dewitte [37-39]). Au vu du nombre restreint des sites et de la taille parfois réduite des échantillons de squelettes intégrés aux recherches des tenants de l'une et l'autre de ces hypothèses, aucune de leurs conclusions ne saurait actuellement être privilégiée ou généralisée sans procéder à de nouvelles études fondées sur un corpus matériel plus conséquent.

Ce constat a motivé la réalisation d'un travail de recherche doctorale [40], dont la présente contribution rapporte les principaux résultats. Ce travail avait une double finalité. D'une part, il visait à caractériser la structure par âge et par sexe d'échantillons ostéoarchéologiques en lien avec des épidémies de peste, ceci afin d'identifier ses éventuelles particularités par rapport à un modèle de mortalité naturelle. D'autre part, il avait pour objectif de qualifier l'état de santé de victimes de la maladie et de le comparer à celui d'individus décédés hors contexte épidémique, afin d'évaluer dans quelle mesure les conditions morbides dont souffraient les membres des populations du passé ont contribué à accroître leur risque de succomber à la peste.

\section{Matériel et méthodes}

\section{Échantillon ostéoarchéologique}


Le corpus ostéologique étudié se compose des restes squelettiques de 1090 individus provenant de six ensembles sépulcraux européens de la fin du Moyen Âge et du début de l'époque moderne (Tableau 1). Cet échantillon intègre 500 squelettes provenant de quatre cimetières de pestiférés, dont deux sont contemporains de la Peste noire et deux autres sont en relation avec des épidémies de la fin du XVIe siècle. Il inclut par ailleurs deux séries ostéoarchéologiques médiévales constituées d'individus morts hors contexte de crises de mortalité majeures, lesquelles ont servi de substrat aux analyses comparatives. La pertinence de l'analyse étant directement dépendante de la qualité des choix opérés quant aux séries investiguées, seuls ont été intégrés à l'étude des sites répondant à des critères de sélection rigoureux - i.e. chronologie bien établie, effectifs de squelettes conséquents, bonne préservation des vestiges squelettiques, contexte de mortalité attesté par des sources historiques ou les résultats d'analyses de paléobiochimie moléculaire. Pour ce qui concerne les sites de comparaison, des ensembles funéraires légèrement antérieurs au début de la deuxième pandémie ont été préférés à des sites de chronologie ultérieure, ceci afin d'assurer l'absence en leur sein d'individus morts de la peste, mais également de s'affranchir des biais pouvant résulter des répercussions à long terme des épidémies sur la structure biologique des populations [41].

\section{Méthodes de l'étude anthropologique}

L'étude des diverses collections a consisté, en premier lieu, en une révision systématique de l'âge au décès et du sexe des individus selon un protocole standardisé. L'estimation de l'âge des sujets immatures s'est fondée en priorité sur le degré de minéralisation dentaire [42,43], ainsi que sur le stade de maturation osseuse pour les individus d'âge post-pubertaire [44]. Ces critères ont été ponctuellement suppléés par la prise en compte des longueurs diaphysaires des os longs pour estimer l'âge d'enfants dépourvus de restes dentaires, de même que celui des périnatals et nourrissons $[45,46]$. Pour les adultes, les processus de maturation tardifs que constituent la fusion de la crête iliaque et la fusion de l'extrémité sternale de la clavicule ont été considérés pour identifier les individus de moins de 30 ans [47]. L'âge au décès de ceux ayant achevé leur maturation squelettique a été estimé d'après le degré de remodelage de la surface sacro-pelvienne iliaque [48]. Enfin, le sexe a été déterminé, pour les seuls sujets 
adultes, d'après les caractéristiques morphologiques et métriques de l'os coxal $[49,50]$ et le dimorphisme extra-pelvien propre à chaque échantillon populationnel [51].

Les données individuelles obtenues ont été utilisées pour caractériser la structure par âge et par sexe des séries de peste, à travers le calcul de différents indices paléodémographiques sex ratio, ratio immatures/adultes, indice de mortalité infantile $\left(\mathrm{D}_{0} / \mathrm{D}_{\text {total }}\right)$, indice de juvénilité (D5-14/D20+) - et la construction de profils de mortalité par âge $[52,53]$. Ces données ont été comparées à un modèle de mortalité naturelle, élaboré sur la base de tables-types jugées représentatives des populations archaïques [54]. Les anomalies identifiées ont été objectivées à l'aide de tests statistiques (test du $\chi^{2}$ d'adéquation pour évaluer les écarts du sex ratio par rapport à une situation d'équilibre, test de comparaison des proportions pour confronter les quotients de mortalité de chaque classe d'âge à leurs valeurs théoriques). Ces mêmes données ont été employées pour évaluer l'adéquation de la composition biologique des échantillons avec la structure d'une population vivante préindustrielle, cette dernière ayant été appréhendée par le biais de modèles théoriques (pyramides des âges d'après [54,55]) ainsi que par des recensements de populations médiévales et modernes [20,56-58].

La deuxième partie de l'étude a consisté en un examen macroscopique systématique des vestiges squelettiques afin de documenter la présence de différents indicateurs osseux et dentaires témoignant de stress biologiques et de maladies (Fig. 1). Les lésions pathologiques prises en compte dans l'étude incluent les hypoplasies linéaires de l'émail dentaire (i.e. défauts de minéralisation de la couronne dus à des stress physiologiques durant l'enfance), les cribra orbitalia (i.e. porosités du toit orbitaire témoins d'anémie ou de processus inflammatoires) et diverses modifications osseuses évocatrices d'infection (remodelages endocrâniens, réactions périostées tibiales et intra-thoraciques). Ces diverses atteintes squelettiques ont été enregistrées selon des méthodes de cotation standardisées, dont le détail est consultable dans l'Annexe 1 (voir Matériel Supplémentaire en ligne), ainsi que dans la thèse de doctorat dont dérive cette contribution [40].

La prévalence de ces différents indicateurs a été calculée pour chaque collection, en tenant compte de l'état de conservation des régions squelettiques qu'ils concernent [59]. La relation entre la présence de lésions et les paramètres biologiques (âge/sexe) a été évaluée à l'aide de tests statistiques non paramétriques (test exact de Fisher, test de Mann-Whitney). Les victimes de la peste et les sujets du groupe de comparaison ont été confrontés en 
recourant à ces mêmes tests ainsi qu'à l'utilisation d'odds ratio communs (ÔR), mesure statistique d'usage courant en épidémiologie qui tient compte dans son mode de calcul des différences de structure biologique et des différences de taille d'échantillons entre les groupes comparés $[59,60]$.

\section{Résultats}

\section{Composition biologique des séries de peste}

L'analyse anthropologique a autorisé une caractérisation détaillée de la structure biologique des collections qui, si elles présentent certaines dissemblances, partagent également de nombreux traits communs. En ce qui concerne le sexe, les résultats rendent compte de fluctuations mineures du sex ratio d'un site à l'autre. Les deux séries de la seconde moitié du XIVe siècle renferment ainsi une part plus importante de femmes, tandis que les hommes sont légèrement plus nombreux dans les deux cimetières de la fin du XVIe siècle (Tableau 2). Pour autant, ces rapports ne diffèrent jamais de manière statistiquement significative de la situation théorique d'équilibre entre sexes.

A contrario, la structure par âge des collections se distingue de manière sensible d'un modèle de mortalité naturelle (Fig. 2). Indépendamment de la chronologie et de l'ancrage géographique des sites dont ils proviennent, les assemblages squelettiques se caractérisent par des anomalies récurrentes, à savoir : (1) une sous-représentation des enfants de moins d'un an ( $\mathrm{p}<0,05$ pour chaque site) ; (2) une proportion des décès anormalement élevée entre 5 et 14 ans ( $<<0,05$ pour chaque site) ; (3) une fréquente surreprésentation des grands adolescents (toutefois non statistiquement significative, exception faite pour le site de Termonde) et des jeunes adultes ( $\mathrm{p}<0,05$ pour les deux séries du XVIe siècle). Ce modèle général de mortalité accepte toutefois certaines variations, la principale résidant dans la proportion des sujets immatures d'âge compris entre 5 et 14 ans, plus élevée pour les deux sites du XVIe siècle.

Les profils de mortalité des séries de peste, s'ils se distinguent donc nettement d'un modèle de mortalité naturelle, présentent au contraire de nettes similitudes avec la structure par âge d'une population vivante archaïque (Tableau 3). Ainsi, la faible proportion des sujets de moins d'un an dans les collections apparaît en parfaite cohérence avec les valeurs 
rapportées dans différents recensements de populations préindustrielles. Il en va de même de la proportion des jeunes adultes, à tout le moins pour les deux séries du XVIe siècle, qui sont celles dont l'état de conservation a autorisé la meilleure quantification de la part des adultes de moins de 30 ans (i.e. les seules pour lesquelles cette proportion peut être évaluée avec fiabilité). La proportion des individus d'âge compris entre 5 à 14 ans (exprimé par l'indice de juvénilité) est également conforme à ce que l'on attend d'une population vivante pour les deux sites du XIVe siècle. Elle est au contraire très nettement supérieure aux valeurs attendues pour les deux sites du XVIe siècle, ce qui suggère une mortalité accrue dans ces classes d'âges.

\section{Caractérisation comparée de l'état sanitaire}

Les patterns lésionnels s'étant révélés relativement homogènes au sein des séries relevant d'un même contexte de mortalité (pour une présentation détaillée des études par sites et des comparaisons inter-sites, voir [40]), les individus ont été regroupés en deux larges échantillons (i.e. victimes de la peste et groupe de comparaison) dans la suite de l'analyse. L'étude comparative menée à ce niveau scalaire d'exploitation des données rend compte de différences notables entre groupes, tant en ce qui concerne la prévalence des lésions que leur distribution selon les paramètres d'âge et de sexe. Les principales spécificités de chaque groupe sont synthétisées ci-après, en considérant tour à tour les différents indicateurs squelettiques pris en compte dans l'analyse.

Les hypoplasies linéaires de l'émail dentaire sont fréquentes dans toutes les séries du corpus, indépendamment du contexte de mortalité, du sexe et de l'âge au décès des individus. Des différences patentes entre groupes sont toutefois apparentes lorsque seules sont considérées les formes d'hypoplasies les plus prononcées (i.e. lésions aisément identifiables à l'œil nu, sans recourt à l'examen à la loupe ou à la palpation à l'aide d'une sonde dentaire). Ces lésions entretiennent dans le groupe de comparaison une forte relation à l'âge au décès, leur prévalence étant significativement plus élevée dans la fraction immature de l'échantillon (Fisher ; $p=0,008$ ). Une telle corrélation n'est au contraire pas observée chez les victimes de la peste, concernées en proportions semblables, quel que soit leur âge. De la même manière, le nombre d'épisodes de stress enregistrés par les dentures est plus important chez les enfants 
que chez les adultes dans le groupe de comparaison (Mann-Whitney ; $p=0,034$ ), tandis qu'il ne diffère pas significativement selon l'âge chez les pestiférés (Mann-Whitney ; p =0,472) (Fig. 3).

Les cribra orbitalia entretiennent une même relation à l'âge dans les deux groupes, y étant significativement plus fréquentes chez les sujets immatures que chez les adultes (Fisher ; $\mathrm{p}<0,01)$. Le déclin de leur prévalence à l'âge adulte pourrait toutefois résulter tout ou partie d'une oblitération des porosités orbitaires au cours de la vie sous l'action du remodelage osseux, invitant à une certaine prudence dans l'interprétation de ce résultat. L'analyse de la fraction immature des échantillons apparaît, en ce sens, plus informative. La fréquence des cribra orbitalia y est significativement plus élevée dans le groupe de comparaison, indépendamment de l'âge des individus ( $\mathrm{OR}=2,08 ; \mathrm{p}=0,038$ ). Par ailleurs, les lésions non remodelées, qui témoignent d'un processus pathologique actif peu de temps avant le décès, sont rares chez les pestiférés. Elles sont significativement plus fréquentes chez les sujets du groupe de comparaison (Fisher ; $p=0,002$ ), ce qui suggère que leurs facteurs causaux eurent une influence plus forte sur le risque de décès hors contexte épidémique.

Les remodelages endocrâniens affectent principalement les sujets immatures. Ils sont, chez ces derniers, significativement plus fréquents dans le groupe de comparaison (ÔR = $3,23 ; \mathrm{p}=0,033)$. Leur prévalence y est forte avant 5 ans et diminue sensiblement après cet âge, attestant d'une mortalité infanto-juvénile accrue chez les individus porteurs de telles lésions (Fig. 4). Les remodelages sont au contraire peu fréquents dans le groupe des victimes de la peste et la relation qu'ils entretiennent avec l'âge y apparaît plus ténue.

À la différence des indicateurs précédents, les réactions périostées augmentent progressivement en fréquence avec l'âge. Ce phénomène vaut toutefois plus particulièrement pour les lésions remodelées et traduit donc vraisemblablement un effet cumulatif au cours de la vie, lié à une plus longue durée d'exposition au risque infectieux [61,62]. Les lésions actives, les plus informatives sur l'état de santé peu avant le décès, sont plus fréquentes chez les sujets adultes du groupe de comparaison (Fisher; $p=0,023$ et $p=0,015$ respectivement pour les lésions tibiales et intra-thoraciques), suggérant, une nouvelle fois, une plus forte influence de leurs facteurs causaux sur le risque de décès hors contexte épidémique. 


\section{Discussion}

\section{Ponction démographique des épidémies de peste}

Les résultats de l'étude paléodémographique témoignent de l'existence d'un schéma de mortalité commun aux quatre séries de peste étudiées. Les anomalies récurrentes qui le caractérisent, mises en évidence par ailleurs pour d'autres sites d'inhumation de pestiférés [35,63-66], tendent à démontrer que la maladie a entraîné aux époques passées une ponction démographique spécifique, qui se distingue nettement de la mortalité naturelle. Si ce modèle général accepte certes quelques variations intersites, il s'agit pour la plupart de fluctuations mineures qui ne saurait être interprétées comme le signe d'une hétérogénéité épidémiologique de la maladie selon les contextes chronologiques ou environnementaux. Seule pourrait éventuellement faire exception la variabilité de représentation des enfants les plus âgés et des adolescents (5-14 ans), qu'il reste toutefois en l'état de cette recherche difficile d'attribuer préférentiellement à un biais d'échantillonnage, à une exposition différentielle à la maladie de diverses franges de populations variant selon les contextes chrono-culturels, ou encore à une évolution temporelle dans l'interaction homme/pathogène.

La mise en perspective des profils de mortalité avec la structure théorique d'une population archaïque a mis en exergue la relative adéquation entre la composition par âge des séries de peste et celle d'une population vivante préindustrielle. À la différence des causes générales de mortalité, qui engendrent des décès plus fréquents au sein des classes d'âge constituées des membres les plus fragiles d'une population (i.e. jeunes enfants, personnes âgées), la peste semble ainsi avoir été à l'origine d'une ponction démographique uniforme sans égard pour l'âge des individus. En l'absence d'anomalies univoques quant aux proportions respectives d'hommes et de femmes victimes de la maladie, le sexe semble également ne pas avoir constitué un facteur déterminant du risque d'y succomber, tout du moins au titre des seules caractéristiques épidémiologiques de la maladie. Les résultats de la présente étude plaident donc en faveur d'une absence de sélectivité de la mortalité par peste vis-à-vis de l'âge ou du sexe des individus, contredisant de la sorte les conclusions de précédents travaux anthropologiques [39,67]. Il convient toutefois de souligner ici les limites interprétatives imposées par les méthodes anthropologiques employées, qui n’autorisent ni la 
caractérisation du sex ratio de la fraction immature des échantillons, ni une analyse fine de la répartition par âge des sujets adultes.

Au-delà de leur apport indéniable à la compréhension de l'épidémiologique de la peste dans le passé, les résultats obtenus confirment l'intérêt du «modèle peste » [36] pour la recherche anthropologique. Au vu de la ponction démographique uniforme associée à la maladie, les séries ostéo-archéologiques issues de cimetières de pestiférés sont en en effet susceptibles de constituer, pour peu que l'effectif des squelettes exhumés soit suffisant, des échantillons représentatifs de la population vivante en un lieu donné, à une époque donnée. Ils apparaissent, à cet égard, comme des substrats d'étude de première importance pour mener des travaux anthropologiques à visées paléoépidémiologique.

\section{État de santé préexistant et mortalité par peste}

L'analyse comparative entre groupes rend compte de nets écarts de prévalences lésionnelles en ce qui concerne les remaniements squelettiques résultant de stress et maladies de l'enfance et les signes osseux d'infections en phase d'état ou de convalescence. Ces résultats convergent pour démontrer que les victimes de la peste jouissaient globalement, avant leur décès, d'un meilleur état de santé que leurs homologues morts hors contexte épidémique. Cette conclusion se doit naturellement d'être considérée avec une circonspection de rigueur, imposée par diverses limites méthodologiques et interprétatives. La première tient à l'information même fournie par les modifications squelettiques étudiées, qui peut s'avérer paradoxale : à l'échelle individuelle, la présence d'indicateurs de stress n'indique pas nécessairement un moins bon état de santé que son absence, cette dernière pouvant signaler indifféremment que les sujets n'ont pas souffert d'épisodes morbides majeurs ou qu'ils en ont été affectés mais sont morts rapidement [68]. À l'échelle populationnelle, les indicateurs pris en compte dans l'étude semblent toutefois pouvoir être considérés comme de bons marqueurs d'un état de santé dégradé, à en juger par la relation inversement proportionnelle qu'ils entretiennent avec l'espérance de vie en contexte de mortalité naturelle. Une seconde limite tient à la pluralité des facteurs étiologiques pouvant intervenir dans la formation d'un même indicateur squelettique. L'analyse conjointe de divers types de lésions est en ce sens primordial, autorisant une interprétation plus robuste des résultats [69,70]. La concordance 
dont ils témoignent dans le cas présent permet ainsi d'exclure que les différences intergroupes soient fortuites. Enfin, d'autres limites tiennent à la nature même des groupes comparés, à savoir des échantillons composites formés de l'agrégat d'individus issus de populations distinctes. Au regard des tendances lésionnelles semblables entre séries relevant d'un même contexte de mortalité, les disparités géographiques ou chronologiques entre collections ne sauraient toutefois être considérées comme des sources de biais majeurs.

En dépit des limites énoncées, les résultats obtenus semblent donc constituer des indices recevables d'une différence d'état de santé entre victimes de la peste et individus morts hors contexte épidémique. Ils tendent à démontrer que les facteurs causaux des indicateurs squelettiques étudiés, s'ils furent d'accoutumé responsables d'une diminution des chances de survie, n'eurent au contraire qu'une influence mineure, si ce n'est nulle, sur le risque de mourir de la peste. Cette conclusion s'oppose à celle d'un précédent travail anthropologique qui avait plaidé l'existence d'un lien entre état de santé préexistant et susceptibilité à l'infection pesteuse [37]. Ces résultats divergents pourraient pour partie tirer leur origine de différences d'approches méthodologiques. Celle retenue par S. DeWitte et J. Wood [37], fondée sur l'utilisation d'un modèle de morbidité et de mortalité supposé décrire le risque relatif de mourir en fonction de la présence ou de l'absence de lésions, souffre selon nous de divers écueils - i.e. emploi d'âges précis non assortis de leur intervalle de confiance, modélisation du risque de développer une lésion comme constant au cours de la vie, non prise en compte du degré de remodelage des lésions. L'interprétation retenue par ces auteurs repose en outre sur l'analyse d'un unique site funéraire, excluant toute possibilité de généralisation en termes d'épidémiologie de la maladie.

Sans exclure de manière définitive que l'état de santé puisse, dans certaines circonstances, avoir contribué à une plus grande létalité chez les sujets infectés ou être corrélé à certains facteurs conditionnant un plus grand risque d'exposition à la maladie (e.g. lieux de vie, activités professionnelles), la présente étude tend à démontrer qu'il n'eut probablement qu'un effet marginal sur le risque d'y succomber. À la lueur des résultats obtenus, l'infection à $Y$. pestis apparaît donc comme non sélective à l'égard de ce paramètre biologique. 


\section{Conclusion}

En fournissant des données inédites sur les caractéristiques biologiques d'un large échantillon de victimes de la deuxième pandémie, la présente étude apporte une contribution significative au débat actuel sur la sélectivité de l'infection pesteuse. Ses résultats contredisent ceux de précédents travaux qui avaient conclu, sur la base de l'analyse d'un unique site, à l'existence d'un risque de décès différentiel selon l'âge, le sexe et l'état de santé préexistant. Sans nier que ces paramètres biologiques puissent dans certains cas avoir contribué à accroître le risque de contracter la maladie ou d'y succomber, les résultats obtenus démontrent le caractère non systématique d'une telle relation, qui ne saurait dès lors être mise sur le compte des caractéristiques épidémiologiques de la maladie. Pris dans leur ensemble, ces résultats tendent à prouver que les épidémies de peste de la fin du Moyen Âge et de l'époque moderne n'eurent aucune prédilection que ce soit pour certaines classes biologiques plutôt que d'autres, frappant indistinctement les individus des deux sexes, de tous âges et de toutes conditions sanitaires.

La poursuite de travaux anthropologique sur d'autres séries de pestiférés et l'élargissement de leur cadre chronologique devrait permettre, à l'avenir, de préciser si ces caractéristiques furent immuables à travers le temps et l'espace ou furent au contraire variables selon les contextes chronologiques et environnementaux. L'étude diachronique de larges ensembles de sites couvrant l'ensemble de la deuxième pandémie serait notamment souhaitable pour évaluer si les populations européennes, immunologiquement naïves vis-à-vis de la maladie à l'arrivée de la Peste noire, acquirent peu à peu à son égard quelque forme de résistance au gré de vagues épidémiques récurrentes. L'analyse de séries ostéologiques en lien avec des épidémies de peste antérieures pourraient, quant à elle, permettre de discuter l'évolution éventuelle de la virulence de l'infection, que laissent par ailleurs envisager certaines différences génétiques entre souches bactériennes responsables des première et deuxième pandémies $[16,17]$.

Remerciements : J'adresse mes sincères remerciements à la Société d'Anthropologie de Paris pour m'avoir fait l'honneur de me décerner le prix de la thèse 2016. Je remercie aussi chaleureusement D. Castex (UMR 5199 - PACEA) qui a supervisé ce travail de recherche. Je 
suis également reconnaissant aux chercheurs et institutions qui m'ont permis l'accès aux collections ostéologiques qui en constituent le matériel d'étude : J. Buckberry (Biological Anthropology Research Centre, Bradford), C. Polet (Institut royal des Sciences naturelles de Belgique, Bruxelles), M. Signoli (UMR 7268 - ADES, Marseille), C. Niel (UMR 6273 CRAHAM, Caen), P. Courtaud (UMR 5199 - PACEA, Bordeaux). Ce travail a bénéficié d'une aide de l'État gérée par l'Agence Nationale de la Recherche au titre du programme Investissements d'Avenir (ANR-10-LABX-52) et d'un support financier du projet Région Aquitaine "Troubles épidémiques : diagnostic, diffusion et usages funéraires" (dir. D. Castex) hébergé par la Maison des Sciences de l'Homme d'Aquitaine. J'adresse enfin mes remerciements aux relecteurs anonymes pour leurs commentaires, qui ont contribué à l'amélioration de la version finale du manuscrit.

\section{Références}

1. Tikhomirov E (1999) Épidémiologie et répartition de la peste. Manuel de la peste : épidémiologie, répartition, surveillance et lutte. Organisation Mondiale de la Santé, Genève, pp 11-41

2. Organisation Mondiale de la Santé (2010) Peste humaine : examen de la morbidité et de la mortalité régionales, 2004-2009. Relevé épidémiologique hebdomadaire (OMS) 85(6):40-45 3. Bacot AW, Martin CJ (1914) Observations of the mechanism of the transmission of plague by fleas. J Hyg 13(Suppl):423-439

4. Gratz N (1999) Rongeurs réservoirs et puces vectrices des foyers naturels de peste. Manuel de la peste : epidémiologie, répartition, surveillance et lutte. Organisation Mondiale de la Santé, Genève, pp 65-100

5. Demeure C, Carniel E (2009) Yersinia pestis. Revue Francophone des Laboratoires 415:33-39

6. Rasmussen S, Allentoft ME, Nielsen K, et al (2015) Early divergent strains of Yersinia pestis in Eurasia 5,000 years ago. Cell 163:571-582

7. Valtueña AA, Mittnik A, Key FM, et al (2016) The stone age plague: 1000 years of persistence in Eurasia. bioRxiv:094243 
8. Biraben J-N (1975) Les hommes et la peste en France et dans les pays européens et méditerranéens. I - La peste dans l'histoire. Mouton, Paris, $455 \mathrm{p}$

9. Biraben J-N, Le Goff J (1969) La peste dans le Haut Moyen Âge. Ann Econ Soc Civilis 6:1484-1510

10. Benedictow OJ (2004) The Black Death, 1346-1353: the complete history. Boydell Press, Woodbridge, $433 \mathrm{p}$

11. Cohn S (2002) The Black Death transformed: disease and culture in early Renaissance Europe. Arnold, Londres, $318 \mathrm{p}$

12. Cohn SK (2008) Epidemiology of the Black Death and successive waves of plague. Med Hist 27(Suppl):74-100

13. Drancourt M, Aboudharam G, Signoli M, et al (1998) Detection of 400-year-old Yersinia pestis DNA in human dental pulp: an approach to the diagnosis of ancient septicemia. Proc Natl Acad Sci USA 95(21):12637-12640

14. Haensch S, Bianucci R, Signoli M, et al (2010) Distinct clones of Yersinia pestis caused the Black Death. PLoS Pathog 6(10):e1001134

15. Bos KI, Schuenemann VJ, Golding GB, et al (2011) A draft genome of Yersinia pestis from victims of the Black Death. Nature 478(7370):506-510

16. Wagner DM, Klunk J, Harbeck M, et al (2014) Yersinia pestis and the plague of Justinian 541-543 AD: a genomic analysis. Lancet Infect Dis 14(4):319-326

17. Feldman M, Harbeck M, Keller M, et al (2016) A high-coverage Yersinia pestis genome from a 6th-century justinianic plague victim. Mol Biol Evol:msw170

18. Spyrou MA, Tukhbatova RI, Feldman M, et al (2016) Historical Y. pestis genomes reveal the european Black Death as the source of ancient and modern plague pandemics. Cell Host Microbe 19(6):874-881

19. Mallet E (1835) Notice sur les anciennes pestes de genève. Ann Hyg Publ Med Leg (série 1) $14: 206-220$

20. Hollingsworth MF, Hollingsworth TH (1971) Plague mortality rates by age and sex in the parish St. Botolph's without Bishopsgate, London, 1603. Popul Stud XXVI(1):131-146

21. Ell SR (1989) Three days in October of 1630: detailed examination of mortality during an early modern plague epidemic in Venice. Rev Infect Dis 11(1):128-139

22. Horrox R (1994) The Black Death. Manchester University Press, Manchester, 364 p 
23. Herlihy D (1997) The Black Death and the transformation of the West (édité par S.K. Cohn). Harvard University Press, Cambridge, $117 \mathrm{p}$

24. Biraben J-N (1976) Les hommes et la peste en France et dans les pays européens et méditerranéens. II - Les hommes face à la peste. Mouton, Paris, $416 \mathrm{p}$

25. Panzac D (1986) Quarantaines et lazarets : l'Europe et la peste d'Orient (XVIIe-XXe siècles). Édisud, Aix-en-Provence, 219 p

26. Bourdelais P (2003) Les épidémies terrassées. Une histoire de pays riches. Éditions de La Martinière, Paris, $247 \mathrm{p}$

27. Barry S, Gualde N (2007) La Peste noire dans l'Occident chrétien et musulman : 13461347/1352-1353. In: Castex D, Cartron I (eds) Épidémies et crises de mortalité du passé, Actes des séminaires (année 2005) de la Maison des Sciences de l'Homme d'Aquitaine. Ausonius Éditions, Études 15, Pessac, pp 193-227

28. Dragomanni FG (1846) Cronica di Matteo Villani. Vol. I. Sansone Coen, Florence, 594 p 29. Costa F (2008) Geraldo Oddone, O. min., ministro generale, patriarca d'Antiochia e vescovo di Catania (1342-48). In: Grisanti N (ed) Francescanesimo e cultura nella provincia di Catania, Atti del convegno di studio, Catania, 21-22 dicembre 2007. Biblioteca Francescana - Officina de Studi Medievali, Palerme, pp 21-102

30. Lemaître H (1906) Chroniques et annales de Gilles le Muisit, abbé de Saint-Martin de Tournai (1272-1352). Librairie Renouard, Paris, 388 p

31. Thompson EM (1889) Chronicon Galfridi le Baker de Swynebroke. Clarendon press, Oxford, $340 \mathrm{p}$

32. Riley HT (1863) Thomae Walsingham, quondam monachi S. Albani, historia anglicana, vol. I (A.D. 1272-1381). Eyre \& Spottiswoode, Londres, 629 p

33. Gransden A (1957) A fourteenth-century chronicle from the Grey Friars at Lynn. Eng Hist Rev 72:270-278

34. Signoli M, Séguy I, Biraben JN, et al (2002) Paleodemography and historical demography in the context of an epidemic: plague in Provence in the eighteenth century. Population 57(6):829-854

35. Gowland RL, Chamberlain AT (2005) Detecting plague: palaeodemographic characterisation of a catastrophic death assemblage. Antiquity 79:146-157 
36. Dutour O, Maczel M, Ardagna Y (2007) Intérêt du modèle peste en paléoépidémiologie. In: Signoli M, Chevé D, Adalian P, et al (eds) Peste : entre épidémies et sociétés. Firenze University Press, Florence, pp 101-108

37. DeWitte SN, Wood JW (2008) Selectivity of Black Death mortality with respect to preexisting health. Proc Natl Acad Sci USA 105(5):1436-1441

38. DeWitte SN (2014) The anthropology of plague: insights from bioarcheological analyses of epidemic cemeteries. The Medieval Globe 1:97-123

39. DeWitte SN (2010) Age patterns of mortality during the Black Death in London, AD 1349-1350. J Archaeol Sci 37(12):3394-3400

40. Kacki S (2016) Influence de l'état sanitaire des populations anciennes sur la mortalité en temps de peste. Contribution à la paléoépidémiologie. Thèse de doctorat en Anthropologie Biologique, Université de Bordeaux, 749 p

41. Paine RR (2000) If a population crashes in prehistory, and there is no paleodemographer there to hear it, does it make a sound? Am J Phys Anthropol 112(2):181-190

42. Moorrees CFA, Fanning EA, Hunt EE (1963) Age variation of formation stages for ten permanent teeth. J Dent Res 42(6):1490-1502

43. Moorrees CFA, Fanning EA, Hunt EE (1963) Formation and resorption of three deciduous teeth in children. Am J Phys Anthropol 21(2):205-213

44. Coqueugniot H, Weaver TD, Houët F (2010) Brief communication: a probabilistic approach to age estimation from infracranial sequences of maturation. Am J Phys Anthropol $142: 655-664$

45. Maresh MM (1970) Measurements from roentgenograms. In: McCammon RW (ed) Human growth and development. Charles C. Thomas, Springfield, pp 157-200

46. Sellier P (1993) L'estimation de l'âge des foetus et des enfants morts en période périnatale : une révision de Fazekas et Kosa (1978). Croissance et vieillissement, $21^{\mathrm{e}}$ colloque du Groupement des Anthropologistes de Langue Française (Bordeaux, 13-15 mai 1993), non publié.

47. Owings-Webb PA, Suchey JM (1985) Epiphyseal union of the anterior iliac crest and medial clavicle in a modern multiracial sample of American males and females. Am J Phys Anthropol 68:457-466 
48. Schmitt A (2005) Une nouvelle méthode pour estimer l'âge au décès des adultes à partir de la surface sacro-pelvienne iliaque. Bull. Mém. Soc. Anthropol. Paris 17:89-101

49. Brůžek J (2002) A method for visual determination of sex, using the human hip bone. Am J Phys Anthropol 117:157-168

50. Murail P, Brůžek J, Houët F, et al (2005) DSP: A tool for probabilistic sex diagnosis using worldwide variability in hip-bone measurements. Bull. Mém. Soc. Anthropol. Paris 17(34):167-176

51. Murail P, Brůžek J, Braga J (1999) A new approach to sexual diagnosis in past populations. Practical adjustments from Van Vark's procedure. Int J Osteoarchaeol 9(1):39-53 52. Sellier P (1996) La mise en évidence d'anomalies démographiques et leur interprétation : population, recrutement et pratiques funéraires du tumulus de Courtesoult. In: Piningre J-F (ed) Nécropoles et sociétés au premier âge du Fer : Le tumulus de Courtesoult (Haute-Saône). DAF, pp 188-202

53. Sellier P (1995) Paléodémographie et archéologie funéraire: les cimetières de Mehrgarh, Pakistan. Paléorient 21(2):123-143

54. Ledermann S (1969) Nouvelles tables-types de mortalité. Institut National d'Études Démographiques, Travaux et documents $\mathrm{n}^{\circ} 53$, PUF, Paris, $260 \mathrm{p}$

55. Coale AJ, Demeny P (1966) Regional model life-tables and stable populations. Princeton University Press, Princeton, $504 \mathrm{p}$

56. Russell JC (1958) Late ancient and medieval population. Trans Am Philos Soc 48(3):1152

57. Séguy I, Pennec S, Tzortzis S, et al (2006) Modélisation de l'impact de la peste à travers l'exemple de Martigues (Bouches-du-Rhône). In: Buchet L, Dauphin C, Séguy I (eds) La paléodémographie. Mémoire d'os, mémoire d'hommes, Actes des 8èmes journées anthropologiques de Valbonne. APDCA, Antibes, pp 323-331

58. Noël R (1967) La population de la paroisse de Laguiole, d'après un recensement de 1691. Ann Demogr Hist 1:197-223

59. Waldron T (2008) Palaeopathology. Cambridge University Press, New Yok, 279 p 60. Klaus HD (2014) Frontiers in the bioarchaeology of stress and disease: cross-disciplinary perspectives from pathophysiology, human biology, and epidemiology. Am J Phys Anthropol 155(2):294-308 
61. Glencross B, Sawchuk L (2003) The person-years construct: ageing and the prevalence of health related phenomena from skeletal samples. Int J Osteoarchaeol 13(6):369-374

62. Grauer AL (1993) Patterns of anemia and infection from medieval York, England. Am J Phys Anthropol 91:203-213

63. Margerison BJ, Knüsel CJ (2002) Paleodemographic comparison of a catastrophic and an attritional death assemblage. Am J Phys Anthropol 119(2):134-143

64. Castex D (2008) Identification and interpretation of historical cemeteries linked to epidemics. In: Raoult D, Drancourt M (eds) Paleomicrobiology: past human infections. Springer-verlag, Berlin, pp 23-48

65. Kacki S, Castex D (2014) La sépulture multiple de la basilique des Saints Martyrs Just et Pastor : bio-archéologie des restes humains. Quaderns d'Arqueologia i Història de la Ciutat de Barcelona 10:180-199

66. Castex D, Kacki S (2016) Demographic patterns distinctive of epidemic cemeteries in archaeological samples. Microbiol Spectr 4(4):PoH-0015-2015

67. DeWitte SN (2010) Sex differentials in frailty in medieval England. Am J Phys Anthropol $143: 285-297$

68. Wood JW, Milner GR, Harpending HC, et al (1992) The osteological paradox: problems of inferring prehistoric health from skeletal samples. Current Anthropology 33(4):343-358

69. Goodman AH, Martin DL (2002) Reconstructing health profiles from skeletal remains. In: Steckel RH, Rose JC (eds) The backbone of history: health and nutrition in the western hemisphere. Cambridge University Press, Cambridge, pp 11-60

70. Wright LE, Yoder CJ (2003) Recent progress in bioarchaeology: approaches to the osteological paradox. J Archaeol Res 11(1):43-70 

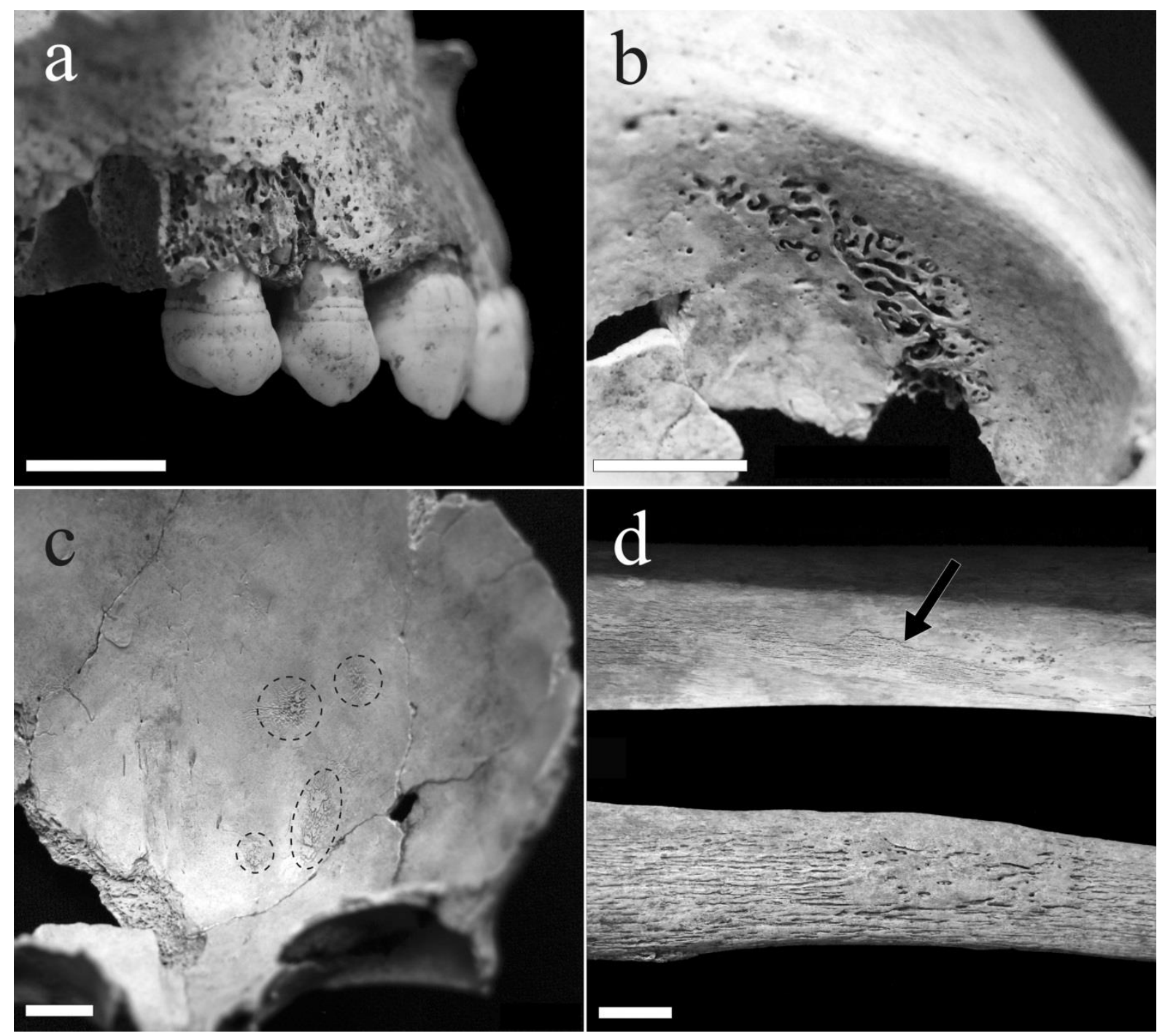

Fig. 1. Indicateurs squelettiques de l'état sanitaire pris en compte dans l'analyse : hypoplasies linéaires de l'émail dentaires (a), cribra orbitalia (b), remodelages endocrâniens (c) et réactions périostées (d). Échelles : $1 \mathrm{~cm} /$ Indicators of health status considered in the study: linear enamel hypoplasia (a), cribra orbitalia (b), endocranial lesions (c), and periosteal reactions $(d)$. Scales: $1 \mathrm{~cm}$. 


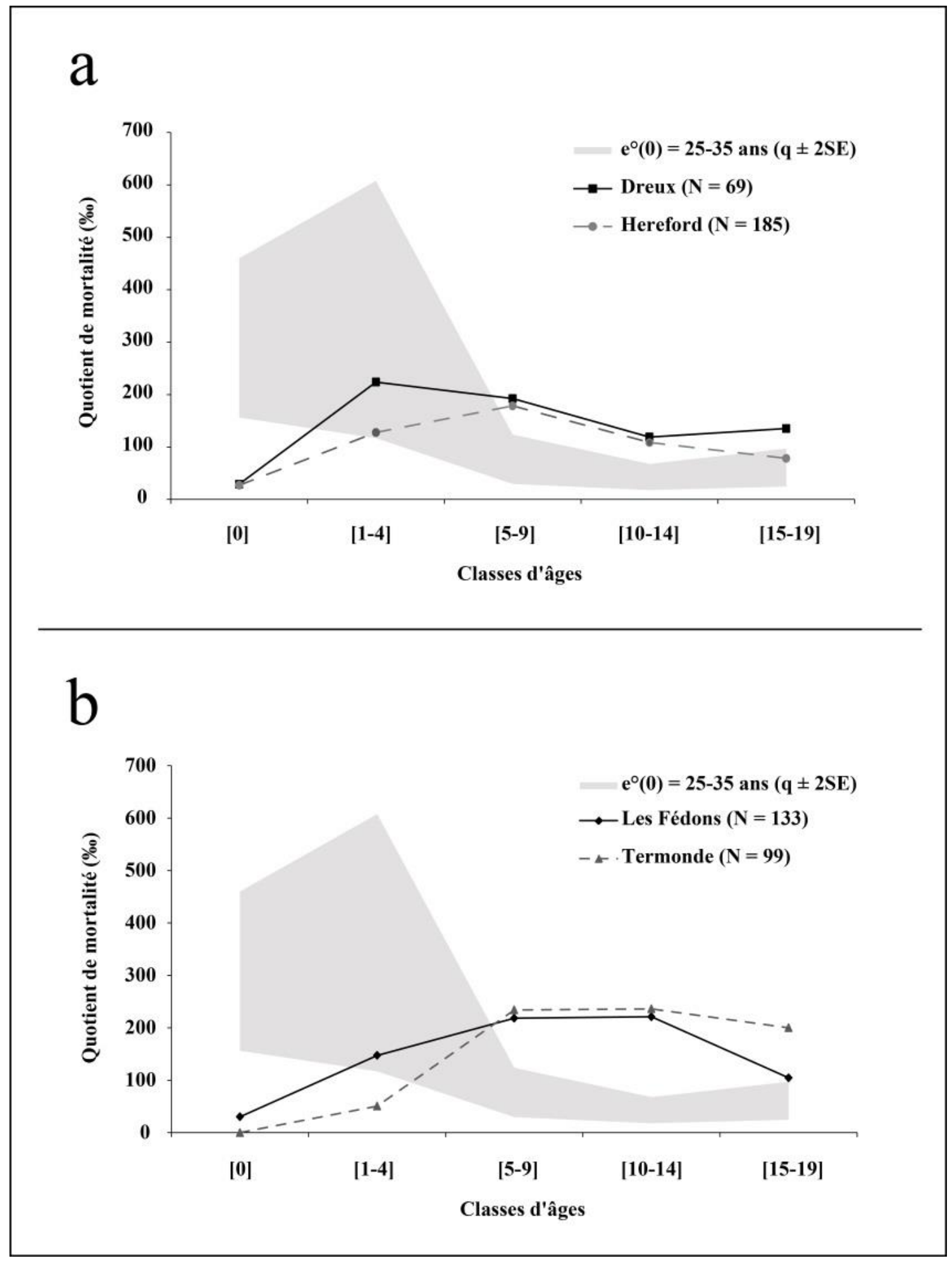

Fig. 2. Profils de mortalité par âge des séries de pestiférés du XIVe siècle (a) et du XVIe siècle (b) comparés aux quotients de mortalité théoriques d'un modèle de mortalité naturelle (d'après Ledermann [54]) / Age-at-death distribution of non-adults from 14th century (a) and $16 t$ century (b) plague cemeteries, compared with the range of theoretical values for nonepidemic mortality (after Ledermann [54]). 


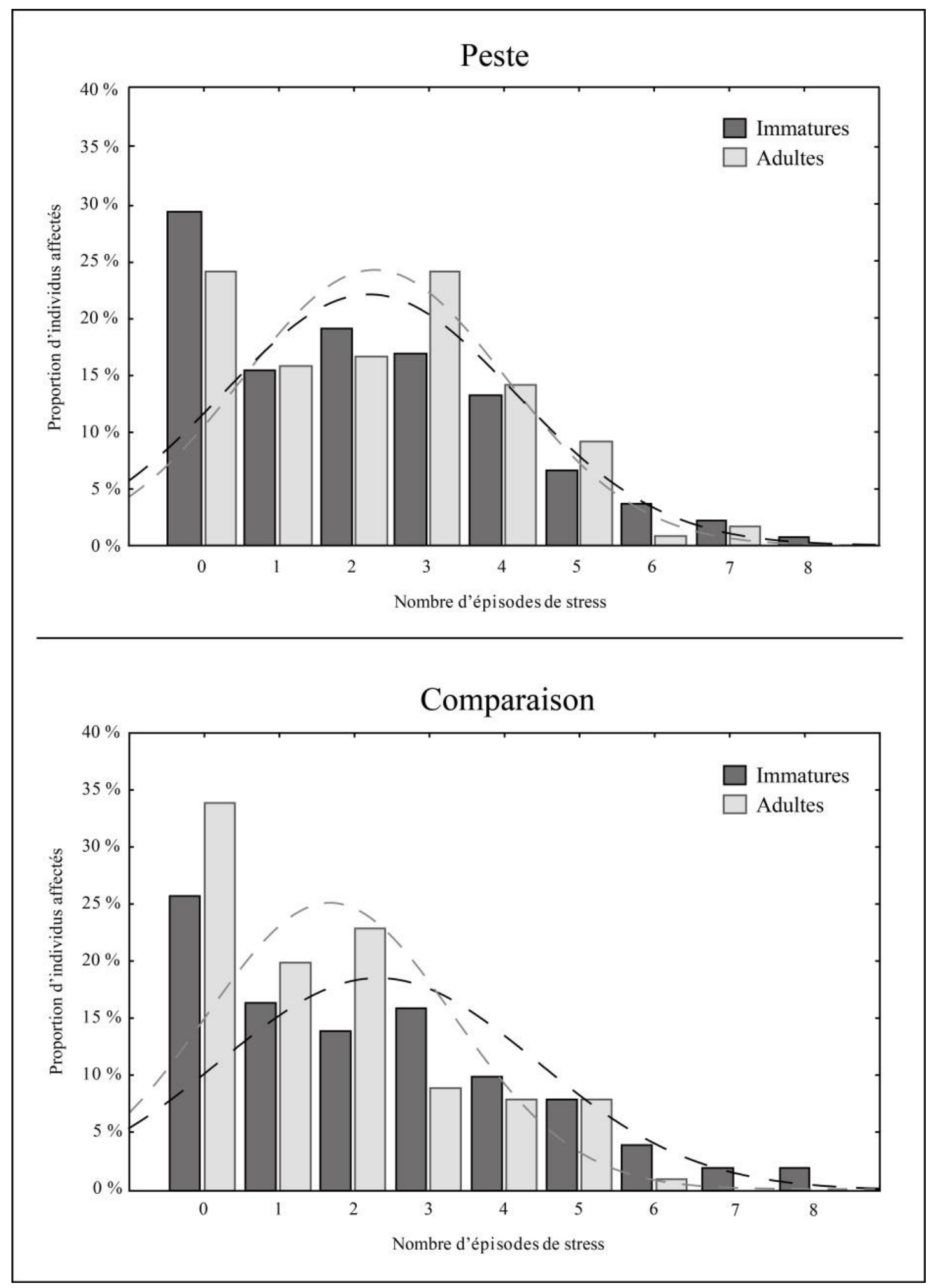

Fig. 3. Nombre d'épisodes de stress enregistrés par les dentures permanentes des sujets immatures et adultes de chaque groupe. Les courbes de Gauss permettent d'apprécier les écarts de distribution selon l'âge au décès / Number of stress episodes per individual, as reconstructed from enamel hypoplasia in permanent teeth. Gauss curves show differences in the number of lesions between non-adults and adults. 


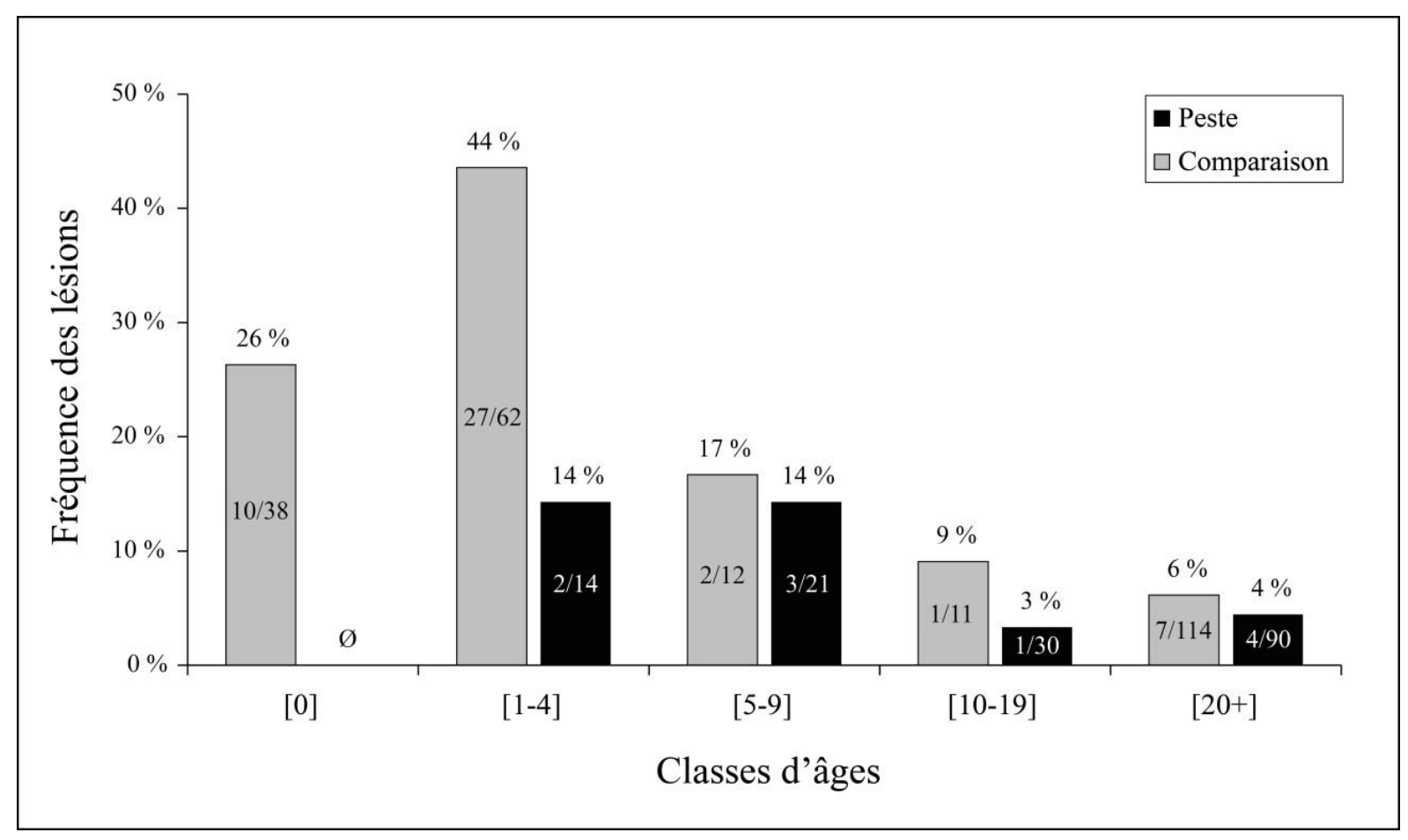

Fig. 4. Prévalence de remodelages endocrâniens en fonction de l'âge chez les victimes de la peste et les sujets du groupe de comparaison $(\varnothing:$ aucune donnée pour cette classe d'âge) / Prevalence of endocranial lesions according to age in the plague victims and in the control group (Ø: no data for this age group). 
Tableau 1. Effectif des sujets étudiés dans chacun des sites du corpus (IM : immatures ; AD : adultes) / Number of skeletons per site in the plague group and in the control group (IM : non-adults; $A D$ : adults).

\begin{tabular}{lllccc}
\hline \multirow{2}{*}{ Groupe } & \multirow{2}{*}{ Site } & \multirow{2}{*}{ Chronologie } & \multicolumn{3}{c}{ Effectifs des sujets étudiés } \\
\cline { 4 - 6 } & & & IM & AD & Total \\
\hline \multirow{3}{*}{ Sites de } & Dreux, Place Métezeau (France) & XIVe s. & 37 & 32 & 69 \\
peste & Hereford Cathedral (Angleterre) & XIVe s. & 79 & 106 & 185 \\
& Lambesc, Les Fédons (France) & XVIe s. & 73 & 60 & 133 \\
& Termonde, Maria Troon (Belgique) & XVIe s. & 55 & 44 & 99 \\
& Sous-total sites de peste & - & 244 & 242 & 486 \\
\hline \multirow{2}{*}{ Sites de } & Toulouse, Saint-Etienne (France) & XIe-XIIIe s. & 161 & 81 & 242 \\
comparaison & Rouen, Notre-Dame (France) & XIe s. & 180 & 182 & 362 \\
& Sous-total sites de comparaison & - & 341 & 263 & 604 \\
\hline \multirow{2}{*}{ Corpus } & Total & - & 585 & 515 & 1090 \\
\hline
\end{tabular}

Tableau 2. Composition sexuelle des séries de peste et résultats des tests statistiques évaluant leur divergence avec une situation théorique d'équilibre du sex ratio $(\mathrm{M}$ : hommes; F : femmes ; I : sujets de sexe indéterminé) / Sexual composition of the skeletal assemblages, and results of statistical comparison with a balanced sex ratio (M: males; F: females; I: unsexed individuals).

\begin{tabular}{|c|c|c|c|c|c|c|c|c|}
\hline \multirow{2}{*}{ Site } & \multirow{2}{*}{$\begin{array}{l}\text { Effectif } \\
\text { adulte }\end{array}$} & \multicolumn{3}{|c|}{ Sexe } & \multirow{2}{*}{$\begin{array}{l}\text { Taux de } \\
\text { diagnose }\end{array}$} & \multirow{2}{*}{$\begin{array}{l}\text { Sex } \\
\text { ratio }\end{array}$} & \multirow{2}{*}{$\chi^{2}$} & \multirow{2}{*}{$\mathbf{p}$} \\
\hline & & M & $\mathrm{F}$ & I & & & & \\
\hline Dreux & 32 & 11 & 15 & 6 & $81 \%$ & $0,73: 1$ & 0,62 & 0,433 \\
\hline Hereford & 106 & 40 & 52 & 14 & $87 \%$ & $0,77: 1$ & 1,56 & 0,211 \\
\hline Les Fédons & 60 & 30 & 26 & 4 & $93 \%$ & $1,15: 1$ & 0,29 & 0,593 \\
\hline Termonde & 44 & 20 & 18 & 6 & $86 \%$ & $1,11: 1$ & 0,11 & 0,746 \\
\hline
\end{tabular}


Tableau 3. Comparaison de divers indices paléodémographiques des séries étudiées avec leurs valeurs théoriques dans des populations vivantes préindustrielles. Les valeurs théoriques sont évaluées par le biais de recensements de populations médiévales et modernes, ainsi qu'à partir de modèles extrapolés de tables-types de mortalité $\left(\mathrm{e}^{\circ}{ }_{0}\right.$ : espérance de vie à la naissance ; r : taux d'accroissement de population) / Palaeodemographic indices in the skeletal assemblages compared with their theoretical values in living pre-industrial populations. Theoretical values are estimated using medieval and post-medieval population censuses, and model life tables ( $e_{0}^{\circ}$ : life expectancy at birth; $r$ : population growth rate).

\begin{tabular}{|c|c|c|c|c|c|c|c|}
\hline \multirow{2}{*}{ Source des données } & \multicolumn{2}{|c|}{$\mathrm{D}_{0} / \mathrm{D}_{\text {total }}$} & \multicolumn{2}{|c|}{$\mathrm{D}_{5-14} / \mathrm{D}_{20+}$} & \multicolumn{2}{|c|}{$\mathrm{D}_{20-29} / \mathrm{D}_{20+}$} & \multirow{2}{*}{ Référence } \\
\hline & $\mathrm{n} / \mathrm{N}$ & $\%$ & $\mathrm{n} / \mathrm{N}$ & ratio & $\mathrm{n} / \mathrm{N}$ & $\%$ & \\
\hline \multicolumn{8}{|l|}{ Séries ostéologiques } \\
\hline Dreux (XIVe s.) & $2 / 69$ & 2,9 & $15 / 32$ & 0,47 & $2 / 32$ & 6,3 & [40] \\
\hline Hereford (XIVe s.) & $5 / 185$ & 2,7 & $42 / 106$ & 0,40 & $18 / 106$ & 17,0 & [40] \\
\hline Les Fédons (XVIe s.) & $4 / 133$ & 3,0 & $43 / 60$ & 0,72 & $17 / 60$ & 28,3 & [40] \\
\hline Termonde (XVIe s.) & $0 / 99$ & 0,0 & $39 / 44$ & 0,89 & $13 / 44$ & 29,5 & [40] \\
\hline \multicolumn{8}{|l|}{ Recensements de populations } \\
\hline St Victor, Marseille (IXe s.) & $29 / 719$ & 4,0 & $134 / 308$ & 0,44 & $106 / 308$ & 34,4 & [56] \\
\hline Pozzuoli (1489) & $47 / 2527$ & 1,9 & $716 / 1132$ & 0,63 & $435 / 1132$ & 38,4 & [56] \\
\hline Sorrento (1561) & $20 / 2918$ & 0,7 & $576 / 1510$ & 0,38 & $535 / 1510$ & 35,4 & [56] \\
\hline St Botolph, Londres (1601) & $210 / 7802$ & 2,7 & $1791 / 4189$ & 0,43 & $1276 / 4189$ & 30,5 & [20] \\
\hline Laguiole (1691) & - & - & $181 / 587$ & 0,31 & $180 / 587$ & 30,7 & [58] \\
\hline Martigues (1720) & $246 / 6069$ & 4,1 & $1241 / 3536$ & 0,35 & $906 / 3536$ & 25,6 & [57] \\
\hline \multicolumn{8}{|l|}{ Modèles théoriques } \\
\hline Ledermann $\left(\mathrm{e}^{\circ}{ }_{0}=25 \mathrm{ans}\right)$ & - & 4,7 & - & 0,34 & - & 27,7 & [54] \\
\hline Ledermann $\left(\mathrm{e}^{\circ}{ }_{0}=35\right.$ ans $)$ & - & 3,0 & - & 0,29 & - & 25,1 & [54] \\
\hline Ouest, niveau $5(\mathrm{r}=0,00)$ & - & 2,8 & - & 0,33 & - & 28,4 & [55] \\
\hline Ouest, niveau $5(\mathrm{r}=0,02)$ & - & 4,6 & - & 0,60 & - & 37,9 & {$[55]$} \\
\hline
\end{tabular}

